JOURNAL OF ETHNOBIOLOGY

AND ETHNOMEDICINE

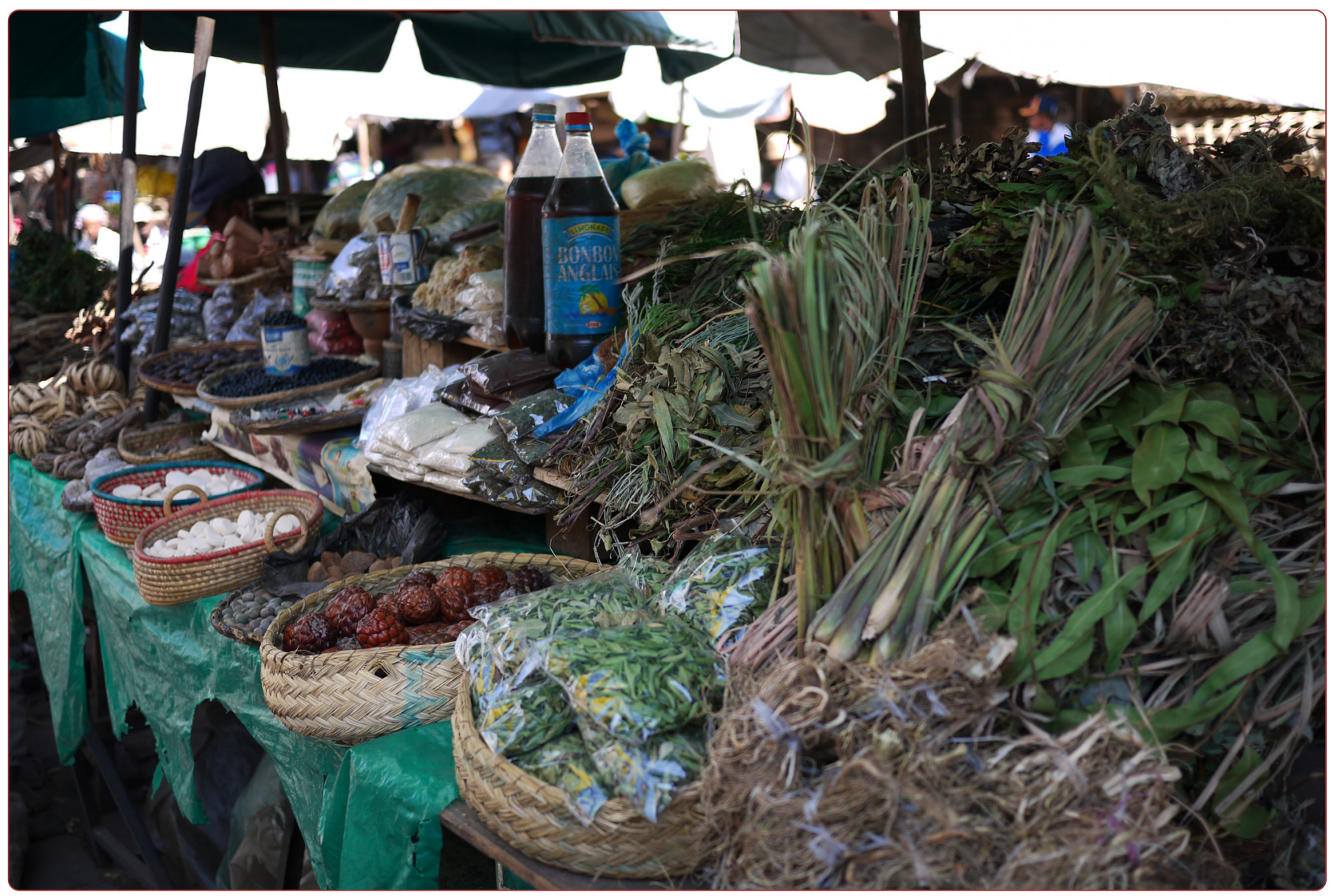

\title{
Medicinal plants sold in the markets of Antananarivo, Madagascar
}

Randriamiharisoa et al. 


\title{
Medicinal plants sold in the markets of Antananarivo, Madagascar
}

\author{
Maria Nirina Randriamiharisoa ${ }^{*}$, Alyse R. Kuhlman², Vololoniaina Jeannoda', Harison Rabarison ${ }^{1}$, \\ Nivo Rakotoarivelo ${ }^{3}$, Tabita Randrianarivony ${ }^{3}$, Fortunat Raktoarivony ${ }^{3}$, Armand Randrianasolo ${ }^{2}$ \\ and Rainer W. Bussmann ${ }^{2}$
}

\begin{abstract}
Background: This study focuses on the large outdoor markets of the capital of Madagascar, Antananarivo. As the largest metropolitan area in Madagascar with a population of nearly two million, the region has great capacity for consumption of medicinal plant remedies despite numerous pharmacies. Medicinal plant use spans all socioeconomic levels, and the diverse metropolitan population allows us to study a wide variety of people who consume these plants for medical purposes. The purpose of this study is to identify and generate a list of medicinal plants sold in the traditional markets with a focus on those collected in the forests around Antananarivo, get an idea of the quantities of medicinal plants sold in the markets around Antananarivo, and assess the economy of the medicinal plant markets.
\end{abstract}

Methods: In order to determine which medicinal plants are most consumed in Antananarivo, ethnobotanical enquiries were conducted in the five main markets of the capital city. Ethnobotanical surveys were conducted with medicinal plant traders, suppliers, harvesters and cultivators, with voucher specimens created from the plants discussed. Trade circuit information was established and the income generated by the trade of some of the species was assessed.

Results: The inventory of the Antananarivo markets resulted in a list of 89 commercialized plant species. Ten of the 89 were mentioned by $60-100 \%$ of vendors. Profitability for vendors is high and competitive with other salaried positions within Antananarivo. Transportation costs are also high and therefore lower profitability for other members in the supply chain.

Conclusions: The markets of Antananarivo have always played a vital cultural role in the lives of urban Malagasy, but our study shows they also play an economic role not only for urban residents but rural harvesters as well. Continued research and monitoring of the non-timber forest products trade in Antananarivo is needed to better understand the impact of trade on the wild plant populations.

Keywords: Madagascar, Urban market, Medicinal plants

\section{Background}

The use of plants for medical treatment and therapy is a practice as old as humanity, dating as far back as the oldest known written documents and found in nearly every known culture [1-3]. Traditional medicine is rich due to the diversity of human groups, languages, and customs, combined with the diversity of ecological regions, leading

\footnotetext{
* Correspondence: randriamiharisoa.maria@yahoo.fr

${ }^{1}$ Department of Plant Biology and Ecology, Faculty of Science, University of Antananarivo, Antananarivo 101 BP 566, Madagascar

Full list of author information is available at the end of the article
}

to innovative plant use and specialized knowledge [4]. The World Health Organization estimates that nearly $80 \%$ of the population in developing countries depends mainly on traditional medicine for the treatment of ailments [5]. The dependence on remedies derived from medicinal plants is particularly important in developing countries where modern medicine is often absent or simply too expensive $[6,7]$. Economic devaluation of the developing countries leads to higher prices of pharmaceuticals and makes medicinal plants and traditional medicine more attractive [8]. Additionally, some prefer traditional medicine for various 
reasons including familiarity, tradition and perceived safety $[9,10]$.

Medicinal plants can be of great importance in the daily lives of those who live near places where they grow, not only for their healing traditions but as a commodity to take to the urban areas where they are not locally found to be sold in the marketplace [11]. Trade of non-timber forest products (NTFP) has been a mainstay for rural economies with a large majority being sourced from wild populations [12]. Rural farmers and residents therefore have a financial interest to not only exploit and develop trade of these natural resources [13], but also to consider conservation measures [14, 15]. The domestic market of medicinal plants of Madagascar is not well documented, and the market for medicinal plants and derivatives only represents a small fraction compared to all internal and external trade of the country [16]. Our study focused on the city of Antananarivo and its medicinal plant markets. As the capital of Madagascar Antananarivo is the largest metropolitan area with a population of nearly 2 million, and the region has great potential for consumption of medicinal plant remedies despite numerous allopathic pharmacies [11]. Medicinal plant-use in Madagascar spans all socioeconomic levels and the diverse metropolitan population allowed to study a wide variety of people using plant products. The objective of this study was to identify and generate a list of medicinal plants sold in the traditional markets with a focus on those collected in the forests around Antananarivo, as well as getting information on the quantities of medicinal plants sold in the markets around Antananarivo, and to assess the economy of the medicinal plant markets. Interviews were started with the vendors at the major markets of Antananarivo, and continued with suppliers wherever possible. We then tried to elucidate who cultivated or harvested plants sourced by the suppliers and finally who held the knowledge of traditional plant medicine for the region.

\section{Methods \\ Study area}

Antananarivo is the capital of Madagascar, the fourth largest island in the world, and centrally located in the highlands at nearly 1,300 meters above sea level [17]. We conducted surveys in five major markets of Antananarivo: the Esplanade Analakely, Petite Vitesse, Pavilion Analakely, Isotry and Andravohangy. These markets were chosen based on the following criteria: market size and popularity, medicinal plant species sold on the premises, and knowledge of vendors regarding the use and sale of medicinal plants. Furthermore, markets in Antananarivo are housed in permanent buildings where vendors occupy permanent booths, which allowed for repeat visits to the same vendor to update lists and conduct further interviews.

\section{Markets}

The medicinal plant market includes two subsectors: the traditional medicinal plant market and the pharmaceutical market. The traditional plant market, known as raokandro, includes plants for public use with little to no processing (dried, raw material). The plants were sold either singularly or as a mix with other plants for a particular treatment. Other types of legal plant markets in Antananarivo are pharmaceutical, cosmetics and aromatherapy shops marked with HOMEOPHARMA and IRMA, selling mostly medicinal plants and medicinal plant products that have undergone extensive modification (liquid extract, cream, ointment). The present study focused on the medicinal plant trade within the raokandro. A variety of actors were involved in the sale of medicinal plants. These included operators, collectors, harvesters, and small retailers. The definitions we followed were taken from the ministerial decree number 2915/87 of 30 June 1987 and the Decree of 17 November 1930 mentioned in Articles 32 and 33 are presented in Table 1.

Table 1 Definition of participants within the herbal market trade scheme. Types of collectors and their role within the trade as defined by the Madagascar government

\begin{tabular}{ll}
\hline Operators & $\begin{array}{l}\text { Persons who legally hold a license or an operating agreement to operate and collect medicinal plants and forest } \\
\text { products to sell or use as raw materials. } \\
\text { Collectors }\end{array}$ \\
& $\begin{array}{l}\text { These are individuals who collect plants from those who harvest in the forest. They are authorized to carry out the } \\
\text { grouping of plants with several collectors. } \\
\text { Harvesters }\end{array}$ \\
Rural harvesters & $\begin{array}{l}\text { These are the persons authorized to conduct harvesting or gathering medicinal plants for commercial purposes } \\
\text { market sellers }\end{array}$ \\
Urban harvesters & $\begin{array}{l}\text { people living in the vicinity of the capital, which also make deliveries to vendors of medicinal plants in the } \\
\text { traditional market of Antananarivo }\end{array}$ \\
Public resellers (vendors) & These are the people who sell plants to the public. Called "tapa-mpivarotra kazo" or "mpivarotra raokandro" in Malagasy.
\end{tabular}




\section{Ethnobotanical surveys}

To gather information about the market of medicinal plants, a series of semi-structured interviews were conducted with traders at the traditional markets (raokandro) of Antananarivo after obtaining oral prior informed consent. Questionnaires were used as a foundation for discussions with the collectors and traders. During market interviews we conducted our survey individually and iteratively [18]. All medicinal species that were discussed with the vendors were also purchased from the vendors at the regular price. Medicinal plants were then identified at the department of Plant Biology and Ecology at the University of Antananarivo and crosschecked with published ethnobotanical and floristic literature where available [19-22]. Plant names follow www. TROPICOS.org. Herbarium vouchers were deposited at the herbaria of Centre National de la Recherche Appliquée au Developement Rural (TEF), Parc de Tsimbazaza (TAN) and Missouri Botanical Garden (MO).

\section{Statistical analysis}

For each medicinal plant a Use Index (UI\%) was calculated to give a ranking of the importance of the use and trade of medicinal species in markets of Antananarivo. The UI\% is calculated from the formula, UI $=(\mathrm{na} / \mathrm{NA}) \mathrm{x}$ 100 , where na is the number of interviewees who cite the species as useful and NA is the totally number of people interviewed [23]. In this case, na represents represent the number of vendors who sell a particular medicinal species. The following formulas were used to calculate the profit margin of the various intermediaries surveyed. For sellers, Bv $=\mathrm{PV}$ - PA where the benefit to vendors $(\mathrm{Bv})$ is the difference between the sale price (PV) and the purchase price (PA). For harvesters (rural and urban), $\mathrm{Bh}=\Sigma \mathrm{R}-\Sigma \mathrm{Ex}$, where the benefit to harvesters $(\mathrm{Bh})$ is the difference between the revenue (R) and expenditure costs (Ex). Profit margin (PM) was calculated with $\mathrm{PM}=\mathrm{B} / \Sigma \mathrm{R}$, based on [23].

\section{Results and discussion}

We interviewed 86 people in the traditional markets of medicinal plants in Antananarivo. Table 2 summarizes the survey sites and the number of informants surveyed.

We were able to identify 89 medicinal plant species from 56 vendors. A list of medicinal plants is presented in Table 3. The actual number of species sold is likely higher than what we were able to identify because of the study's limited duration [24]. Furthermore, vendors spoke only about plants that at the time of the interview were available in their stalls. Other plants might be sold at other times, but if they were not available for purchase the sellers did not mention them.

Among the medicinal species available at the major markets of the city of Antananarivo, we encountered
Table 2 Market sites and number of informants surveyed

\begin{tabular}{llll}
\hline Market & $\begin{array}{l}\text { Number of } \\
\text { vendors }\end{array}$ & $\begin{array}{l}\text { Rural } \\
\text { harvesters }\end{array}$ & $\begin{array}{l}\text { Intermediaries or } \\
\text { Urban harvesters }\end{array}$ \\
\hline $\begin{array}{l}\text { Esplanade } \\
\text { Analakely }\end{array}$ & 9 & 0 & 3 \\
Petite Vitesse & 21 & 15 & 7 \\
Andravoahangy & 21 & 5 & 0 \\
Pavilion Analakely & 2 & 0 & 0 \\
Isotry & 3 & 0 & 0 \\
Total & 56 & 20 & 10 \\
Total interviewed & 86 & & \\
\hline
\end{tabular}

nine plant part used: leaves (73\%), bark (7\%), stems $(5 \%)$, roots $(5 \%)$, entire plant $(4 \%)$, fruit $(2 \%)$, tuber (2\%), flower (1\%), other (1\%). (Fig. 1) Leaves were by far the most common plant material used, followed by bark. While leaves and bark were often well represented in other studies, only $50 \%$ of the combined total in our study were leaves and bark, similar to in Sierra Leone [25]. These most common health complaints treated with plants were hepatitis, kidney stones, asthenia, wounds, coughs and gastroenteritis (Fig. 2).

\section{Most traded medicinal species}

Table 4 lists the ten most traded species in the markets, including the Use Index calculated for each of these species, which varied from $61 \%$ to $100 \%$. Prices are typically the main economic indicators about the supply and demand for a product, with higher prices indicating species with higher demand and lower supply. However, we found that the organization of economic actors within the regional medicinal plant trade was also a determinant of prices, often affecting the price based on who and how the species was sourced. Vendors bought their plants from rural harvesters, urban harvesters, and collectors, which is a common trade pattern found in other parts of Africa as well [26]. Increased number of intermediaries before a species reaches the sellers increased the price on the market. Two commercial channels could be distinguished: a short circuit, when harvesters moved to Antananarivo to be closer to the markets in order to sell their products directly themselves, and a long circuit, consisting of a long chain of intermediaries the products passed through before reaching sellers in Antananarivo (Fig. 3). The purchase price of medicinal plants varied widely depending on the species, but we found that prices were constant for a given species.

However, product price increased with each change of hands as transportation costs or other fees incurred. As found in other parts of the world, the amount of time, energy and resources needed to transport medicinal plants to the market was considered extremely high [27]. In addition, the price also fluctuated depending on the 
Table 3 List of medicinal plants sold at the Antananarivo medicinal markets. Scientific name, vernacular name, plant part used, disease treated and voucher number [MTR= Randriamiharisoa, Maria T.] for all 89 plants identified at the Antananarivo Markets. Use citations were compared with Madagascar ethnobotany published literature: [1] Boiteau P, Allorge- Boiteau L, 1993; [2] Samyn, JM, 1999; [3] Gurib-Fakim A, Brendler T, 2004

\begin{tabular}{|c|c|c|c|c|c|}
\hline Scientific name & Vernacular name & Part used & Application & Uses cited in literature & Voucher number \\
\hline \multicolumn{6}{|l|}{ Acanthaceae } \\
\hline $\begin{array}{l}\text { Avicennia marina } \\
\text { (Forssk.) Vierh. }\end{array}$ & Afiafy & Leaf & Stomach ulcer & & MTR142 \\
\hline Justicia sp. & Belohalika & Leaf & Neuralgia & & MTR190 \\
\hline \multicolumn{6}{|l|}{ Amaranthaceae } \\
\hline $\begin{array}{l}\text { Cyathula uncinulata } \\
\text { (Schrad.) Schinz }\end{array}$ & Tangogo & Leaf & $\begin{array}{l}\text { Stomach ulcer, hepatitis, } \\
\text { diabetes, cardiac problems }\end{array}$ & & MTR163 \\
\hline \multicolumn{6}{|l|}{ Anacardiaceae } \\
\hline $\begin{array}{l}\text { Anacardium } \\
\text { occidentale L. }\end{array}$ & Mahabibo & Leaf & $\begin{array}{l}\text { Diabetes, hemorrhoids, } \\
\text { stomach ulcer, allergies, } \\
\text { hepatitis, wounds, } \\
\text { incontinence, anorexia }\end{array}$ & & MTR127 \\
\hline $\begin{array}{l}\text { Rhus taratana (Baker) } \\
\text { H. Perrier }\end{array}$ & Andriambavimahery & Leaf & Wounds, stomach ulcer & & MTR174 \\
\hline \multicolumn{6}{|l|}{ Apiaceae } \\
\hline $\begin{array}{l}\text { Centella asiatica (L.) } \\
\text { Urb. }\end{array}$ & Talapetraka & Entire plant & Stomach ulcer, wounds & $\begin{array}{l}\text { Wounds }{ }^{3} \text {, skin eczema }{ }^{3}, \text { accesses }^{3} \text {, } \\
\text { conjunctivitis }\end{array}$ & MTR138 \\
\hline \multicolumn{6}{|l|}{ Apocynaceae } \\
\hline $\begin{array}{l}\text { Catharanthus lanceus } \\
\text { (Bojer ex A. DC.) } \\
\text { Pichon }\end{array}$ & Vonenina & Root & Cancer & $\begin{array}{l}\text { Diuretic }{ }^{2}, \text { purgative }^{2}, \text { vermifuge }^{2}, \\
\text { sores }^{2}\end{array}$ & MTR161 \\
\hline $\begin{array}{l}\text { Catharanthus roseus } \\
\text { (L.) G. Don }\end{array}$ & Vonenina & Root & Cancer, appetite suppressant & $\begin{array}{l}\text { Hypotensive }^{1} \text {, antidepressant }{ }^{1}, \\
\text { antitumoral }^{1} \text {, } \text { purgative }^{2} \text {, diabetes }{ }^{2} \text {, } \\
\text { appetite suppressant }^{2}, \text { vermifuge }^{3} \text {, } \\
\text { diarrhea }^{3} \text {, dysentery }{ }^{3}\end{array}$ & MTR162 \\
\hline Cynanchum sp. & Vahamavo & Leaf & Asthenia, erectile dysfunction & & MTR191 \\
\hline Pentopetia sp. & Tandrokosy & Leaf & Cough, hepatitis, neuralgia & & MTR189 \\
\hline \multicolumn{6}{|l|}{ Araliaceae } \\
\hline $\begin{array}{l}\text { Schefflera bojeri } \\
\text { (Seem.) R. Vig. }\end{array}$ & Tsingila & Leaf & Stomach ulcer, hepatitis & & MTR143 \\
\hline Schefflera sp. & Ramadio & Leaf & Neurasthenia, back pain & & MTR144 \\
\hline \multicolumn{6}{|l|}{ Asteraceae } \\
\hline $\begin{array}{l}\text { Brachylaena ramiflora } \\
\text { (DC.) Humbert }\end{array}$ & Ramanjavona & Leaf & Asthenia, stomach ulcer, & & MTR173 \\
\hline Cynara scolymus L. & Artichaut & Leaf & Stomach ulcer, hepatitis & & MTR192 \\
\hline $\begin{array}{l}\text { Distephanus } \\
\text { polygalifolius (Less.) } \\
\text { H. Rob. \& B. Kahn }\end{array}$ & Ninginingina & Leaf & $\begin{array}{l}\text { Syphilis, neuralgia, back pain, } \\
\text { stomach ulcerm, hepatitis, } \\
\text { albumin, incontinence }\end{array}$ & & MTR136 \\
\hline Emilia citrina DC. & Tsiotsiona & Leaf & Asthenia, anorexia & & MTR202 \\
\hline $\begin{array}{l}\text { Helichrysum faradifani } \\
\text { Scott- Elliot }\end{array}$ & Haihalala & Leaf & $\begin{array}{l}\text { Gonorrhea, cough, asthenia, } \\
\text { fever, stomach ulcer, hepatitis }\end{array}$ & & MTR159 \\
\hline $\begin{array}{l}\text { Helichrysum } \\
\text { gymnocephalum } \\
\text { (DC.) Humbert }\end{array}$ & Rambiazina & Leaf & $\begin{array}{l}\text { Stomach ulcer, cough, wound, } \\
\text { severe headache }\end{array}$ & $\begin{array}{l}\text { Headaches }{ }^{1} \text {, bronchitis } \\
\text { heartburn } \\
\text { he }^{2} \text { upset stomach } \\
\text { fever }^{2} \text {, diarrhea } \\
\text { rheumatism }^{3}, \text { gout }^{3}\end{array}$ & MTR160 \\
\hline $\begin{array}{l}\text { Inulanthera brownii } \\
\text { (Hochr.) Källersjö }\end{array}$ & Kelimavitrika & Leaf & $\begin{array}{l}\text { Immune system of children, } \\
\text { erectile dysfunction, stiffness }\end{array}$ & & MTR128 \\
\hline \multirow[t]{2}{*}{$\begin{array}{l}\text { Psiadia altissima (DC.) } \\
\text { Drake }\end{array}$} & Sakatavilotra & Leaf & Cough, wound, diarrhea & $\begin{array}{l}\text { Fever }^{3} \text {, abdominal pain } \\
\text { antiseptic } \\
\text {, toothache } \\
\text {, } \text { boils }^{3}\end{array}$ & MTR220 \\
\hline & Ramijaingy & Leaf & & & MTR201 \\
\hline
\end{tabular}


Table 3 List of medicinal plants sold at the Antananarivo medicinal markets. Scientific name, vernacular name, plant part used, disease treated and voucher number [MTR= Randriamiharisoa, Maria T.] for all 89 plants identified at the Antananarivo Markets. Use citations were compared with Madagascar ethnobotany published literature: [1] Boiteau P, Allorge- Boiteau L, 1993; [2] Samyn, JM, 1999; [3] Gurib-Fakim A, Brendler T, 2004 (Continued)

Senecio canaliculatus

Bojer ex DC.

Vernonia

appendiculata Less.

Ambiaty

Bignoniaceae

Jacaranda

mimosifolia D. Don

Phyllarthron

bojeranum DC.

Symphytum orientale Konsody ou Maseza Leaf

L.

Cactaceae

Cereus triangularis (L) Tsilo

Haw.

Root Kidney stones, urinary tract

problems, syphilis, gonorrhea

Astenia, erectile dysfunction, stomach ulcer

madagascariensis

Mandravasarotra Bark

Danguy

Celastraceae

Mystroxylon aethiopicum (Thunb.)

Fanazava

Loes.

Combretaceae

combretum

coccineum (Sonn.)

Lam.

Terminalia catappa L. Atafana

Commelinaceae

Commelina

Nifinakanga

madagascarica C.B.

Clarke

Crassulaceae

Kalanchoe prolifera R. Sodifafana

Hamet

Tamenaka Fruit

Leaf

Leaf

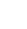

Leaf Neurasthenia

Stem

Emphysema, back pain

subsp.

Fonjozoro

Neuralgia, hepatitis, albumin, erectile dysfunction, back pain, urinary tract problems, stomach ulcer, hypertension, immune deficiency

madagascariensis

(Willd.) Kük.

Droseraceae

Drosera

madagascariensis DC.

Mahantanando

Leaf

Conjunctivitis, enurensis

Ebenaceae

Diospyros sp.

Bois de rose

Bark

Cysticercosis, intestinal

parasites, taxoplasmosis,

regulation, allergies

Intestinal parasites

astroenteritis emphysema, diabetes, albumin

MTR193

MTR145

Stomach pain ${ }^{3}$, colic $^{3}$, analgesic ${ }^{3}, \quad$ MTR194 indigestion ${ }^{3}$, stimulant $^{3}$, cough $^{3}$, dysentery ${ }^{3}$

Fatigue $^{3}$, neuralgia ${ }^{3}$, purgative ${ }^{3}$, vertigo $^{3}$

MTR126

Anthelmintic ${ }^{3}$, liver problems ${ }^{3}$

MTR200

Astringent $^{3}$, sudorific ${ }^{3}$, dysentery ${ }^{3}$

MTR188

MTR176

Boils $^{3}$, furuncles ${ }^{3}$, wounds ${ }^{3}$,

MTR186 rheumatism ${ }^{3}$

MTR146

Equisetaceae

.


Table 3 List of medicinal plants sold at the Antananarivo medicinal markets. Scientific name, vernacular name, plant part used, disease treated and voucher number [MTR= Randriamiharisoa, Maria T.] for all 89 plants identified at the Antananarivo Markets. Use citations were compared with Madagascar ethnobotany published literature: [1] Boiteau P, Allorge- Boiteau L, 1993; [2] Samyn, JM, 1999; [3] Gurib-Fakim A, Brendler T, 2004 (Continued)

\begin{tabular}{llll}
\hline Equisetum sp. & Tsitoatoana & Leaf & $\begin{array}{l}\text { Constipation, urinary tract } \\
\text { problems }\end{array}$
\end{tabular}

Euphorbiaceae

Ricinus communis L. Tanantanamanga Leaf

Fabaceae

\begin{tabular}{|c|c|c|c|}
\hline $\begin{array}{l}\text { Caesalpinia bonduc } \\
\text { (L.) Roxb. }\end{array}$ & Vatolalaka & Fruit & Hemorrhoids, appendicitis \\
\hline $\begin{array}{l}\text { Phylloxylon } \\
\text { xylophylloides (Baker) } \\
\text { Du Puy, Labat \& } \\
\text { Schrire }\end{array}$ & Arahara & Leaf & $\begin{array}{l}\text { Hepatitis, urinary tract } \\
\text { problems, pharyngitis }\end{array}$ \\
\hline $\begin{array}{l}\text { Senna septentrionalis } \\
\text { (Viv.) H.S. Irwin \& } \\
\text { Barneby }\end{array}$ & Anjanajana & Leaf & $\begin{array}{l}\text { Immune system children, } \\
\text { gastroenteritis }\end{array}$ \\
\hline $\begin{array}{l}\text { Senna occidentalis (L.) } \\
\text { Link }\end{array}$ & Tsotsorinangatra & Stem & $\begin{array}{l}\text { Syphilis, gonorrhea, prostate } \\
\text { tumor, hypertension, hepatitis, } \\
\text { rheumatism }\end{array}$ \\
\hline Tamarindus indica $\mathrm{L}$. & Voamadilo & Leaf & $\begin{array}{l}\text { Constipation, gastroenteritis, } \\
\text { wounds }\end{array}$ \\
\hline
\end{tabular}

Gentianaceae

Tachiadenus

longifolius Scott-

Tapabatana

Leaf

Asthenia, hemorrhoids, wounds, intestinal parasites, cold

Galactagogue ${ }^{1,2}$, purgative ${ }^{1,2}$ laxative ${ }^{1,2}$, intestinal worms ${ }^{1}$, tapeworm ${ }^{1}$, headache ${ }^{2}$, rheumatism ${ }^{2}$, dental cavities $^{2}$ wounds ${ }^{2}$, fevers ${ }^{2}$
MTR164

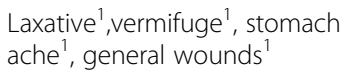
hilsenbergii R. Br.

\section{Hydrostachys} stolonifera Baker

Hypericaceae

Harungana

madagascariensis

Harongana

Leaf

Wounds, asthma, cough, stomach ulcer, hepatitis, gastroenteritis, albumin, allergies, insomnia

$\begin{array}{llll}\text { Psorospermum sp. } & \text { Todihazo } & \text { Stem } & \text { Scabies, leprosy } \\ \begin{array}{l}\text { Psorospermum } \\ \text { ferrovestitum Baker }\end{array} & \text { Andriambolamena } & \text { Leaf } & \begin{array}{l}\text { Female infertility, abortifacient, } \\ \text { stomach ulcer, hypertension, } \\ \text { intestinal parasites }\end{array}\end{array}$

Scabies ${ }^{1,2}$, stomach ache ${ }^{1}$,

flatulence ${ }^{1}$, anticatarrhal ${ }^{1,2}$, bladder infections $s^{2}$, syphilis ${ }^{2}$, menstruation regulation ${ }^{2}$, fever ${ }^{2}$, wounds ${ }^{2}$, diarrhea $^{2,3}$, hemorrhoids ${ }^{2}$, skin diseases $^{3}$

stomach ulcer, hypertension,

Lamiaceae

\begin{tabular}{|c|c|c|c|}
\hline $\begin{array}{l}\text { Ocimum gratissimum } \\
\mathrm{L} \text {. }\end{array}$ & Romba & Leaf & $\begin{array}{l}\text { Severe headache, albumin, } \\
\text { wounds, abortifacient, cold, low } \\
\text { calcium, dental problems }\end{array}$ \\
\hline $\begin{array}{l}\text { Tetradenia riparia } \\
\text { (Hochst.) Codd }\end{array}$ & Borona & Leaf & Cough, wounds, hepatitis \\
\hline \multicolumn{4}{|l|}{ Lauraceae } \\
\hline $\begin{array}{l}\text { Cinnamomum } \\
\text { camphora (L.) J. Presl }\end{array}$ & Ravitsara & Leaf & $\begin{array}{l}\text { Stomach ulcer, hepatitis, } \\
\text { abortifacient, jaundice, }\end{array}$ \\
\hline
\end{tabular}

Digestion ${ }^{3}$, chest complaints ${ }^{3}$, diarrhea ${ }^{3}$, vomiting ${ }^{3}$, anticatarrh ${ }^{3}$, antiseptic $^{3}$

Fevers $^{3}$, rheumatism ${ }^{3}$, abortifacient ${ }^{3}$ 
Table 3 List of medicinal plants sold at the Antananarivo medicinal markets. Scientific name, vernacular name, plant part used, disease treated and voucher number [MTR= Randriamiharisoa, Maria T.] for all 89 plants identified at the Antananarivo Markets. Use citations were compared with Madagascar ethnobotany published literature: [1] Boiteau P, Allorge- Boiteau L, 1993; [2] Samyn, JM, 1999; [3] Gurib-Fakim A, Brendler T, 2004 (Continued)

hypertension, appendicitis, rheumatism

Loganiaceae

Anthocleista

madagascariensis

Landemy

Leaf

Baker

Lycopodiaceae

Lycopodium sp.

Karakaratoloha

Leaf

Meliaceae

Azadirachta indica A. Nimo Juss.

\section{Cedrelopsis grevei \\ Baill. \\ Neobeguea \\ mahafaliensis J.-F. \\ Leroy \\ Molluginaceae}

Mollugo nudicaulis

Lam.

Aferotany

Moraceae

Ficus reflexa Thunb.

Morus alba L.

Nonoka

Voaroihazo

Tanterakala

Embelia concinna

Baker

Myrtaceae

Eucalyptus citriodora Hook.

Eucalyptus sp.

Kininimpotsy

Syzygium cumini (L.)

Skeels

Nymphaeaceae

Nymphaea sp.

Betsimilana

Leaf

Leaf

Ludwigia octovalvis (Jacq.) P.H. Raven

Orchiaceae

Vanilla

madagascariensis

Rolfe

Pedaliaceae

Uncarina sp.

Farehitra

Leaf

Acne

Female infertility, abortifacient albumin, painful menstruation

Emphysema

Erectile dysfunction, asthenia

Stem

Poaceae malaria, constipation, abdominal colic, severe headache

Hepatitis, hypertension,

Asthenia, diabetes, albumin,

rheumatism, pelvic pain, boils, hepatitis, kidney stones, burns,

Asthenia, erectile dysfunction neurasthenia, back pain

Asthenia, erectile dysfunction'

Hepatitis, gastroenteritis,

Low calium, anorexia

Intestinal parasites, erectile dysfunction
Stomach ulcer, diarrhea, gastroenteritis, epilepsy constipation, high cholesterol wounds, albumin, hemorrhoids

Fever $^{1,2}$, dysentery ${ }^{1,2}$, emetic $^{1,2}$, laxative ${ }^{1,2}$

MTR149

MTR157

MTR124

MTR141

MTR183

MTR209

MTR206

MTR210

MTR211

MTR131

Nose bleeds ${ }^{3}$, diarrhea ${ }^{3}$ malnourishment ${ }^{3}$

Aphrodisiac $^{1}$

Fandrotrarana 
Table 3 List of medicinal plants sold at the Antananarivo medicinal markets. Scientific name, vernacular name, plant part used, disease treated and voucher number [MTR= Randriamiharisoa, Maria T.] for all 89 plants identified at the Antananarivo Markets. Use citations were compared with Madagascar ethnobotany published literature: [1] Boiteau P, Allorge- Boiteau L, 1993; [2] Samyn, JM, 1999; [3] Gurib-Fakim A, Brendler T, 2004 (Continued)

\begin{tabular}{|c|c|c|c|c|c|}
\hline \multicolumn{6}{|l|}{$\begin{array}{l}\text { Cynodon dactylon (L.) } \\
\text { Pers. }\end{array}$} \\
\hline $\begin{array}{l}\text { Imperata cylindrica } \\
\text { (L.) Raeusch. }\end{array}$ & Fakatenina & Root & Kidney stones & & MTR182 \\
\hline Zea mays $L$. & Volokatsaka & Silk & $\begin{array}{l}\text { Urinary tract problems, } \\
\text { hepatitis, kidney stones }\end{array}$ & & MTR156 \\
\hline \multicolumn{6}{|l|}{ Pteridaceae } \\
\hline $\begin{array}{l}\text { Adiantum capillus- } \\
\text { veneris } L \text {. }\end{array}$ & Ampanga & Leaf & Allergies, cough & $\begin{array}{l}\text { Respiratory problems }{ }^{1} \text {, diuretic }{ }^{1} \text {, } \\
\text { chickenpox }^{1} \text {, measles }\end{array}$ & MTR207 \\
\hline \multicolumn{6}{|l|}{ Ranunculaceae } \\
\hline $\begin{array}{l}\text { Clematis mauritiana } \\
\text { Lam. }\end{array}$ & Farimafy & Leaf & $\begin{array}{l}\text { Stomach ulcer, hepatitis, } \\
\text { erectile dysfunction }\end{array}$ & $\begin{array}{l}\text { Antiasthmatic }{ }^{3}, \text { rheumatism } \\
\text {, } \\
\text { cough }^{3}, \text { bronchitis }^{3}, \text { abdominal } \\
\text { pains }^{3}\end{array}$ & MTR179 \\
\hline \multicolumn{6}{|l|}{ Rubiaceae } \\
\hline Oldenlandia sp. & Ahipody & Leaf & Scabies, leprosy & & MTR218 \\
\hline Paederia foetida $\mathrm{L}$. & $\begin{array}{l}\text { Vahamaibo, } \\
\text { laingomaimbo }\end{array}$ & Leaf & $\begin{array}{l}\text { Dental issues, wound, stomach } \\
\text { ulcer, gastroenteritis }\end{array}$ & $\begin{array}{l}\text { Diuretic }^{1,3}, \text { diaphoretic }^{1}{ }^{1} \\
\text { purgative }^{1} \text {, skin issues }{ }^{1,3}, \text { ulcers }^{1,} \\
\text { boils }^{3} \text {, venereal diseases }{ }^{3}, \text { bladder } \\
\text { issues }^{3}, \text { gastric pains }\end{array}$ & MTR123 \\
\hline $\begin{array}{l}\text { Pauridiantha } \\
\text { paucinervis (Hiern) } \\
\text { Bremek. }\end{array}$ & Tamirova & Leaf & $\begin{array}{l}\text { Stomach ulcer, hepatitis, } \\
\text { hypertension, urinary tract } \\
\text { problems, rheumatism, malaria, } \\
\text { albumin, diabetes }\end{array}$ & & MTR153 \\
\hline \multicolumn{6}{|l|}{ Rutaceae } \\
\hline $\begin{array}{l}\text { Toddalia asiatica (L.) } \\
\text { Lam. }\end{array}$ & Fanala simba & Elaf & Syphilis, gonorrhea & $\begin{array}{l}\text { Malaria }{ }^{3} \text {, digestive complaints } \\
\text { fever }^{3}, \text { cholera }^{3} \text {, diarrhea } \\
\text { rheumatism }\end{array}$ & MTR181 \\
\hline \multicolumn{6}{|l|}{ Salicaceae } \\
\hline $\begin{array}{l}\text { Homalium parkeri } \\
\text { Baker }\end{array}$ & Hazomby & Bark & Dental issues & & MTR140 \\
\hline \multicolumn{6}{|l|}{ Salviniaceae } \\
\hline Azolla sp. & Ramilamina & Lower & Cardiac arrest & & MTR170 \\
\hline \multicolumn{6}{|l|}{ Smilacaceae } \\
\hline Smilax anceps Willd. & Avotra & Leaf & Gastroenteritis, abdominal colic & $\begin{array}{l}\text { Varicose veins }{ }^{3}, \text { eczema }^{3} \text {, liver } \\
\text { disorders }\end{array}$ & MTR180 \\
\hline \multicolumn{6}{|l|}{ Solanaceae } \\
\hline $\begin{array}{l}\text { Brugmansia candida } \\
\text { Pers. }\end{array}$ & Datroa & Leaf & Epilepsy, paraplegia & & MTR152 \\
\hline Physalis peruviana $\mathrm{L}$. & Voanantsindrana & Leaf & $\begin{array}{l}\text { Rheumatism, urinary tract } \\
\text { problems, syphilis, stomach } \\
\text { ulcer, hepatitis }\end{array}$ & $\begin{array}{l}\text { Eat berries before physical } \\
\text { exertion }^{1} \text {, diuretic }{ }^{1,3}, \text { kidney }^{2} \\
\text { stones }^{1} \text {, rheumatism }{ }^{1} \text {, abscess } \\
\text { liver disease } \\
\text { palpitations }\end{array}$ & MTR137 \\
\hline $\begin{array}{l}\text { Solanum } \\
\text { mauritianum Scop. }\end{array}$ & Seva & Leaf & Hepatitis, wound & $\begin{array}{l}\text { General disinfectant }{ }^{1}, \text { Stomach } \\
\text { ulcers }^{2}\end{array}$ & MTR151 \\
\hline \multicolumn{6}{|l|}{ Stilbaceae } \\
\hline Nuxia capitata Baker & Valanirana & Leaf & Gastroenteritis, asthenia, cough & & MTR169 \\
\hline \multicolumn{6}{|l|}{ Urticaceae } \\
\hline $\begin{array}{l}\text { Urera acuminata } \\
\text { (Poir.) Gaudich. ex }\end{array}$ & Sampy vato & Leaf & $\begin{array}{l}\text { Kidney stones, abortifacient, } \\
\text { hepatitis, stomach ulcer }\end{array}$ & $\begin{array}{l}\text { Irritant to skin and eyes }{ }^{3} \text {, } \\
\text { childbirth }^{3}\end{array}$ & MTR133 \\
\hline
\end{tabular}


Table 3 List of medicinal plants sold at the Antananarivo medicinal markets. Scientific name, vernacular name, plant part used, disease treated and voucher number [MTR = Randriamiharisoa, Maria T.] for all 89 plants identified at the Antananarivo Markets. Use citations were compared with Madagascar ethnobotany published literature: [1] Boiteau P, Allorge- Boiteau L, 1993; [2] Samyn, JM, 1999; [3] Gurib-Fakim A, Brendler T, 2004 (Continued)

\begin{tabular}{|c|c|c|c|c|c|}
\hline \multicolumn{6}{|l|}{ Verbenaceae } \\
\hline Lantana camara L. & Randriaka & Leaf & Hemorrhage, hypertension & & MTR155 \\
\hline \multicolumn{6}{|l|}{ Xanthorrhoeaceae } \\
\hline $\begin{array}{l}\text { Aloe macroclada } \\
\text { Baker }\end{array}$ & Vahona & Leaf & Cancer, allergies, acne, fungus & & MTR139 \\
\hline $\begin{array}{l}\text { Dianella ensifolia (L.) } \\
\text { DC. }\end{array}$ & Erana & Leaf & $\begin{array}{l}\text { Intestinal parasites, } \\
\text { constipation, back pain, } \\
\text { gonorrhea }\end{array}$ & $\begin{array}{l}\text { Eczema }^{3} \text {, dysentery }{ }^{3}, \text { stomach } \\
\text { pains }^{3}\end{array}$ & MTR154 \\
\hline \multicolumn{6}{|l|}{ Zingiberaceae } \\
\hline Zingiber sp. & Tamotamo & Tuber & Cough & & MTR135 \\
\hline $\begin{array}{l}\text { Zingiber officinale } \\
\text { Roscoe }\end{array}$ & Sakamalao & Tuber & Cough & & MTR134 \\
\hline
\end{tabular}

customer's apparent wealth and the type of market (i.e.: tourist handicraft market). Medicinal plants were often supplied from a collector two to four times a week, while some species were only delivered once a month or once a year (in the case of plants came from other provinces of Madagascar). Urban harvesters could afford to bring small amounts of plants (a basket or box) as they sold their products almost daily. Table 5 summarizes the types of providers and delivery frequency by type of market.

Local markets worldwide are a thriving business for both rural and urban dwellers, with a steady demand for medicinal plants. To understand the possible benefits for rural harvesters, several factors needed to be taken into account: 1) the cost of transporting goods 2) the frequency of deliveries to the Antananarivo markets 3) the quantity and value of the species transported to the market. Transport costs from rural areas of Antananarivo depended greatly upon the state of the road and mode of transportation and varied from $\$$ 0.45 - \$ 1.34 per person transporting plants. The most

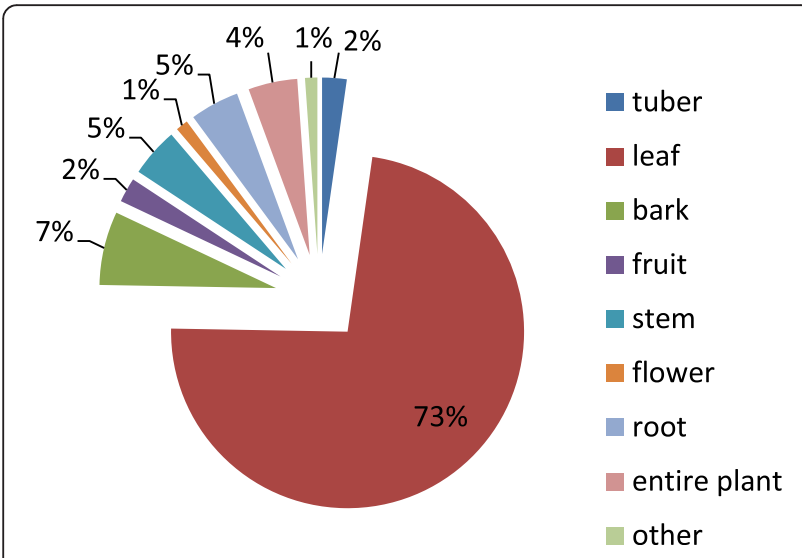

Fig. 1 Plant parts most commonly sold common mode of transport was carrying plant products "on their backs", or by hand, from the rural areas to the city market, with costs ranging from $\$ 0.08-\$$ 0.15 per bag. Overall, transportation costs to deliver the goods to the vendors of medicinal plants in the major markets of the city of Antananarivo ranged anywhere from \$ 3.39 - \$ 8.57 per week. If four bags of medicinal plants (which was the standard weekly amount per vender) were sold at a price of $\$ 4$ - \$ 5 per bag, earnings were $\$ 12$ - \$ 20 a week. The profit margin ranged from $40 \%-81 \%$.

\section{Case study: Pauridiantha paucinervis and Mystroxylon aethiopicium}

To further analyze the trade value of the medicinal plants in Antananarivo, we used the most used single species, Pauridiantha paucinevris, and a species that was present in most of the mixtures, Mystroxylon aethiopicium for closer analysis.

In the market, Pauridiantha paucinervis was sold packaged in a sealed, labeled bags. We found that package was uniform in all markets. Collectors sold this product to vendors for an average of $\$ 0.06$ per package, and the frequency of deliveries was based on fluctuating demand in the markets. The selling price of the product in the market ranged from \$ $0.08-\$$ 0.17. Thus, the selling price of this product was double or even triple compared to its purchase price. According to our surveys vendors sold an average of six bags of $P$. paucinervis each day. Thus, the average earnings for the sale of $P$. paucinervis amounted to $\$$ 0.50 per day, and the monthly earnings could be upwards of $\$ 22.50$.

Mystroxylon aethiopicium was sold at \$ 0.10 - \$ 0.20 per package, but this species was only rarely sold alone, but rather was packaged with other herbs to form a tea 


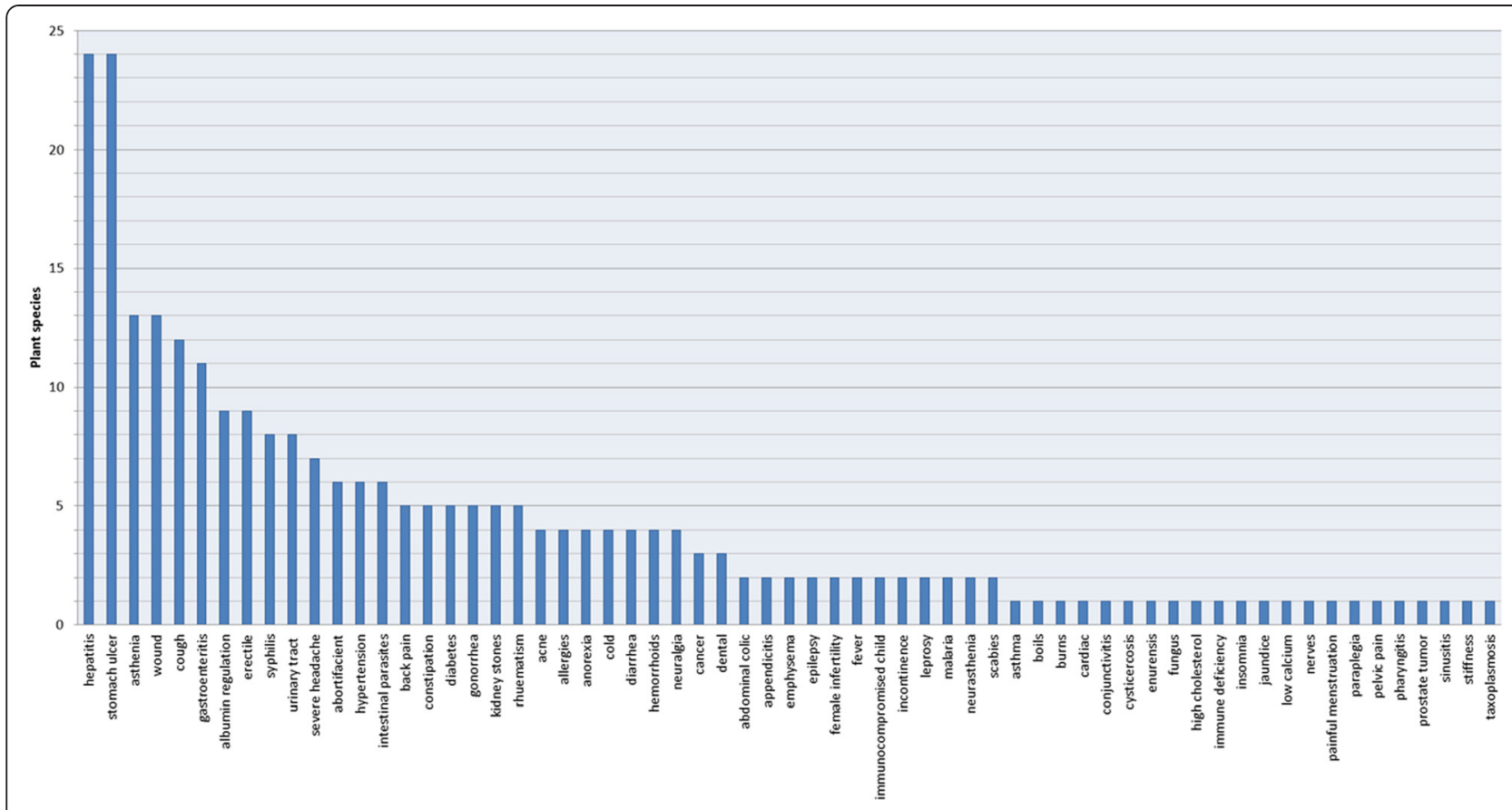

Fig. 2 Number of plant species sold for specific ailments

to treat specific ailments. Sellers bought from collectors once a week, and the order quantity, depending heavily on supply and demand, was often irregular. The purchase price of this species from suppliers was \$ $0.03-$ $\$ 0.30$, depending on volume. The profit margin of sales was $100 \%$ to $150 \%$ if the plant was sold alone, and even higher if it was combined with other herbs. In the latter case, the sale price varied according to the type of disease and also the amount needed for treatment.
Vendors sold an average of 10 packets of $M$. aethiopicium a day, yielding an average of $\$ 0.30$. The average monthly income for a vendor selling $M$. aethiopicium was about $\$ 10$. Therefore, the combined sale of only $P$. paucinervis and $M$. aethiopicium averaged a monthly gross income of $\$ 25$. Considering that the professional monthly minimum wage guarantee in Madagascar is $\$ 25$, the medicinal plant trade can be considered lucrative. However, given the limited amount of time, and

Table 4 Use index calculated for the most traded species and their treatment associations

\begin{tabular}{|c|c|c|c|c|}
\hline Family & Scientific name & Vernacular name & Application & Use index \\
\hline Rubiaceae & $\begin{array}{l}\text { Pauridiantha paucinervis (Hiern) } \\
\text { Bremek. }\end{array}$ & Tamirova & $\begin{array}{l}\text { Stomach ulcer, hepatitis, high blood pressure, urogenital diseases, } \\
\text { rheumatism, malaria, edema, diabetes }\end{array}$ & $100 \%$ \\
\hline Meliaceae & Cedrelopsis grevei Baill. & Katrafay & Asthenia, erectile dysfunction, back pain & $100 \%$ \\
\hline Meliaceae & $\begin{array}{l}\text { Neobeguea mahafaliensis J.-F. } \\
\text { Leroy }\end{array}$ & Andy & Asthenia, erectile dysfunction & $82 \%$ \\
\hline Cactaceae & Cereus triangularis (L.) Haw. & Tsilo & Kidney stones, dysuria, anuria, syphilis, gonorrhea & $78 \%$ \\
\hline Fabaceae & Senna occidentalis (L.) Link & Tsotsorinangatra & $\begin{array}{l}\text { Syphilis, gonorrhea, enlarged prostate, high blood pressure, } \\
\text { rheumatism, hepatitis }\end{array}$ & $70 \%$ \\
\hline Lamiaceae & Ocimum gratissimum L. & Romba & $\begin{array}{l}\text { Intense headache, edema, wounds, repeated miscarriages, cold, } \\
\text { hypocalcemia, dental pain }\end{array}$ & $65 \%$ \\
\hline Boraginaceae & Symphytum orientale L. & Konsody & Stomach ulcer, hepatitis & $65 \%$ \\
\hline Asteraceae & $\begin{array}{l}\text { Cynara cardunculus subsp. } \\
\text { flavescens Wiklund }\end{array}$ & Artichaut & Stomach ulcer, hepatitis & $64 \%$ \\
\hline Asteraceae & $\begin{array}{l}\text { Distephanus polygalifolius (Less.) } \\
\text { H. Rob. \& B. Kahn }\end{array}$ & Ninginingina & $\begin{array}{l}\text { Syphilis, neuralgia, back pain, stomach ulcer, hepatitis, edema, } \\
\text { enuresis }\end{array}$ & $61 \%$ \\
\hline Urticaceae & $\begin{array}{l}\text { Urera acuminata (Poir.) Gaudich. } \\
\text { ex Decne. }\end{array}$ & Sampivato & Kidney stones, repeated miscarriages, hepatitis, stomach ulcer & $61 \%$ \\
\hline
\end{tabular}




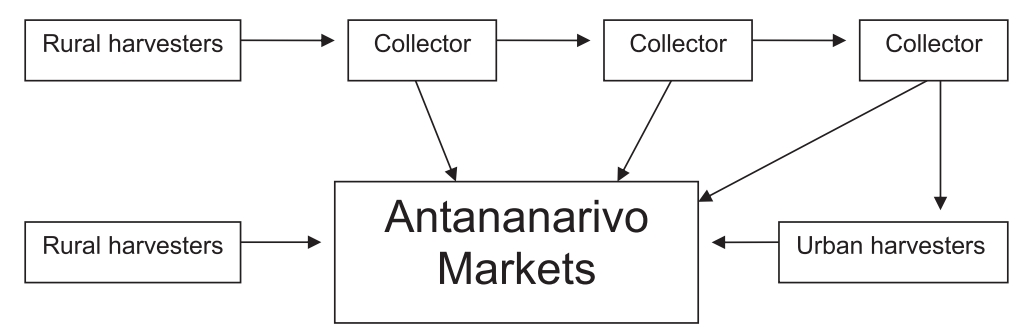

Fig. 3 Market chain of medicinal plants in sold in Antananarivo

limited number of interviews, we could not elucidate the exact quantity of plant material sold in the markets.

\section{Conclusions}

Market studies of non-timber forest products (NFTP) have in the past focused mostly on rural economies and export markets. Recently, increased interest in the domestic marketplace has resulted in more data about economic value of NFTP in the domestic medicinal plant trade. It is difficult to quantify the number of medicinal plants that circulate in the markets of a city like Antananarivo, because this number is highly dependent on market dynamics, which can be quite irregular even for a single plant species. But our estimates show that the sale of medicinal plants in the domestic market provided income for all players - vendors, collectors and harvesters - allowing them to supplement or fully supply their annual income. The impact of these urban traditional markets on the urban and rural economy can be substantial [28]. This booming business has real implications for conservation concerns, which should be researched further to fully explore the impact of the medicinal plant trade on the ecological well-being of the forests where the plants are sourced. Further research and monitoring of the Antananarivo markets will also be invaluable to chart the sustainable use of wild natural resources.

Table 5 Suppliers and frequency of deliveries at each market site

\begin{tabular}{|c|c|c|c|c|}
\hline \multirow[t]{2}{*}{ Market } & \multicolumn{3}{|c|}{ Frequency of delivery } & \multirow[t]{2}{*}{ Transportation } \\
\hline & $\begin{array}{l}\text { Rural } \\
\text { harvesters }\end{array}$ & $\begin{array}{l}\text { Urban } \\
\text { harvesters }\end{array}$ & Collection & \\
\hline Andravoahangy & $\begin{array}{l}2 \text { times a } \\
\text { week }\end{array}$ & Daily & $3 x /$ month & By foot \\
\hline Isotry & Irregular & Daily & Irregular & By foot \\
\hline Petite vitesse & $\begin{array}{l}4 \text { times a } \\
\text { week }\end{array}$ & Daily & $\begin{array}{l}\text { 1-2/ } \\
\text { week }\end{array}$ & By foot \\
\hline $\begin{array}{l}\text { Esplanade } \\
\text { Analakely }\end{array}$ & Irregular & Daily & Irregular & By foot \\
\hline
\end{tabular}

\section{Competing interests}

The authors declare that they have no competing interests.

\section{Authors' contributions}

All authors designed the study and contributed to writing the manuscript. MNR conducted the interviews and completed the data analysis. All authors read and approved the final manuscript.

\section{Acknowledgements}

We would like to thank the Missouri Botanical Garden and the Plant Biology and Ecology Department at the University of Antananarivo for their support and cooperation while carrying out this research. We thank our supervisors for their valuable advice, encouragement and methodological guidelines. We also thank the vendors in all of the markets of Antananarivo for freely giving their time and knowledge.

\section{Author details}

'Department of Plant Biology and Ecology, Faculty of Science, University of Antananarivo, Antananarivo 101 BP 566, Madagascar. ${ }^{2}$ William L. Brown Center, Missouri Botanical Garden, PO Box 299, St. Louis, MO 63166-0299, USA. ${ }^{3}$ Missouri Botanical Garden, Madagascar Research and Conservation Program, Antananarivo 101 BP 3391, Madagascar.

Received: 13 June 2014 Accepted: 17 July 2015

Published online: 28 July 2015

\section{References}

1. Cragg GM, Newman DJ. Natural products: a continuing source of novel drug leads. Biochim Biophys Acta. 2013;1830(6):3670-95.

2. Fowler MW. Plants, medicines and man. J Sci Food Agric. 2006;86:1797-804.

3. Randimbivololona F. Research, valorization and exploitation of biological resources for medicinal purposes in the Malagasy Republic (Madagascar) J Ethnopharmacol. 1996;51:195-200.

4. Schultes RE. The Importance of Ethnobotany in Environmental Conservation. Am J Econ Sociol. 1994;53:202-6.

5. Cunningham AB. African Medicinal Plants: Setting Priorities at the Interface between Conservation and Primary Health Care. People and Plants Working Paper 1. Amsterdam, The Netherlands: Elsevier; 1993.

6. Randrianarivelojosia M, Rasidimanana VT, Rabarison H, Cheplogoi PK, Ratsimbason M, Mulholland DA, et al. Plants traditionally prescribed to treat tazo (malaria) in the eastern region of Madagascar. Malar J. 2003;2:25.

7. Novy JW. Medicinal plants of the eastern region of Madagascar. J Ethnopharmacol. 1997;55:119-26.

8. Ndoye O, Ruiz Perez M, Eyebe A. The markets of non-timber forest products in the humid forest zone of Cameroon. Rural Development Forestry Network 1998, Network Paper 22c. Center for International Forestry Research, Yaoundé, Cameroon.

9. Sheldon JW, Balick MJ, Laird SA. Medicinal plants: can utilization and conservation coexist? Adv Econ Bot. 1997:12:1-104.

10. van Andel T, Carvalheiro LG. Why Urban Citizens in Developing Countries Use Traditional Medicines: The Case of Suriname. Evid Based Complement Alternat Med. 2013;2013:687197.

11. Ticktin T. The ecological implications of harvesting non-timber forest products. J Appl Ecol. 2004;41:11-21.

12. Hamilton A. Medicinal Plants and Conservation: Issues and Approaches. London: WWF UK report; 2003. 
13. Augustino S, Gillah PR. Medicinal Plants in Urban Districts of Tanzania: Plants, Gender Roles and Sustainable Use. Int Forest Rev. 2005;7:44-51.

14. Ros-Tonen M. The role of non-timber forest products in sustainable tropical forest management. Holz als Roh-und Werkstoff. 2000;58(3):196-201.

15. Arnold MJE, Ruiz Perez M. Can non-timber forest products match tropical forest conservation and development objectives? Ecol Econ. 2001;39:437-47.

16. World Health Organization. International Union for Conservation of Nature and Natural Resources. Geneva: World Wide Fund for Nature; 1993.

17. United Nations Human Settlements Programme. Madagascar: Profil Urbain D'Antananarivo. Nairobi: United Nations Human Settlement Program; 2012

18. Ramamonjisoa BS. Méthodes d'enquêtes : manuels à l'usage des praticiens. Antananarivo: Manuel forestier n 11 du Département Eaux et Forêts, École Supérieure des Sciences Agronomiques; 1996.

19. Boiteau P, Allorge-Boiteau L. Plantes médicinales de Madagascar: Cinquante- huit plantes médicinales utilisées sur le marché de Tananarive (Zoma) à Madagascar. Paris: Karthala; 1993.

20. Samyn JM. Plantes utiles des hautes terres de Madagascar. Antananarivo: Graphoprint; 1999

21. Schatz G. Generic Tree Flora of Madagascar. Kew: Royal Botanic Gardens; 2001

22. Gurib-Fakim A, Brendler T. Medicinal and Aromatic Plants of Indian Ocean Islands: Madagascar, Comoros, Seychelles, and Mascarenes. Stuttgart, Germany: medpharm GmbH Scientific Publishers; 2004.

23. Lance K, Kremen C, Raymond I. Extraction of forest Products: quantitative of a park and buffer zone and long-term monitoring. Antananarivo: Report to Park Delimitation Unit, WCS/PCDIM; 1994. p. 549-63.

24. Williams V, Witkowski TF, Balkwill K. The use of incidence-based species richness estimators, species accumulation curves and similarity measures to appraise ethnobotanical inventories from South Africa. Biodivers Conserv. 2007;16:2495-513.

25. Jusu A, Cuni Sanchez A. Economic importance of the medicinal plant trade in Sierra Leone. Economic Botany. 2013;67:299-312.

26. Jusu A, Cuni Sanchez A. Medicinal Plant Trade in Sierra Leone: Threats and Opportunities for Conservation. Economic Botany. 2014;68:16-29.

27. Bussmann RW, Sharon D. Markets, Healers, Vendors, Collectors: The Sustainability of Medicinal Plant Use in Northern Peru. Mt Res Dev. 2009;29:128-34.

28. Shackleton S, Shanley P, Ndoye O. Invisible But Viable: Recognising Local Markets for Non-Timber Forest Products. Int Forest Rev. 2007;9(3):697-712.

\section{Submit your next manuscript to BioMed Central and take full advantage of:}

- Convenient online submission

- Thorough peer review

- No space constraints or color figure charges

- Immediate publication on acceptance

- Inclusion in PubMed, CAS, Scopus and Google Scholar

- Research which is freely available for redistribution

Submit your manuscript at www.biomedcentral.com/submit 